\title{
Chimney Endografting for Zone 2 Thoracic Endovascular Aortic Repair Using The Reorientation Technique
}

\author{
1) Department of Interventional Radiology, Yokohama Nanbu Hospital, Japan \\ 2) Department of Interventional Radiology, Yokohama Rosai Hospital, Japan \\ 3) Department of Cardiovascular Surgery, Yokohama Rosai Hospital, Japan \\ 4) Department of Radiology, Yokohama Rosai Hospital, Japan
}

Yuya Koike ${ }^{122}$, Kazuma Date ${ }^{3)}$, Borbala Kiss ${ }^{4)}$

\begin{abstract}
The purpose of this report was to describe the reorientation of the chimney graft technique to downsize brachial artery access during thoracic endovascular aortic repair and thus preserve left subclavian artery flow. In the case described herein, the chimney graft was advanced not from the brachial or axillary artery, but from the common femoral artery, over a brachiofemoral pull-through wire. The chimney graft was then turned out into the ascending aorta by balloon dilatation via percutaneous brachial access ("reorientation"). Despite the use of a large-diameter chimney graft, the chimney technique with percutaneous brachial access was successfully performed using the reorientation technique.
\end{abstract}

Key words: thoracic endovascular aortic repair, chimney technique

(Interventional Radiology 2020; 5: 89-93)

\section{Introduction}

The chimney technique involves placement of stents in the side branches of the aorta alongside the main endovascular stent graft [1, 2]. Thoracic endovascular aortic repair (TEVAR) with the chimney technique to maintain supraaortic branch perfusion provides a safe, minimally invasive alternative for patients with thoracic aortic disease involving the supra-aortic branches, for whom conventional surgery is considered to be high-risk $[3,4]$. Chimney grafts require vascular access using an appropriate 6- to 12-Fr introducer sheath according to the size of the graft. The percutaneous approach limits the size of the vascular access. However, the access used in the chimney technique can be downsized if the chimney graft is delivered and deployed via the femoral approach followed by balloon angioplasty with a percutaneous approach involving the supra-aortic branches (the reorientation technique).
We herein describe the reorientation the chimney graft technique to downsize brachial artery access during zone 2 TEVAR and thus preserve left subclavian artery (LSA) flow.

\section{Case Report}

An 81-year-old man was admitted to our hospital because of multiple saccular aneurysms of the distal aortic arch and descending aorta up to $5.5 \mathrm{~cm}$ in diameter (Figure 1A). He was asymptomatic, and there was no evidence of mycotic aneurysms. He had hypertension, hyperlipidemia, renal impairment, claudication, and had suffered a recent myocardial infarction. Therefore, the patient was deemed to be high risk for conventional surgery. As the lesion was associated with a short proximal landing zone (approximately $5 \mathrm{~mm}$ ) and spinal cord ischemia due to long descending aorta coverage, zone 2 TEVAR with the chimney technique was planned to preserve LSA flow. The new proximal neck length created by the chimney technique was $25 \mathrm{~mm}$. 


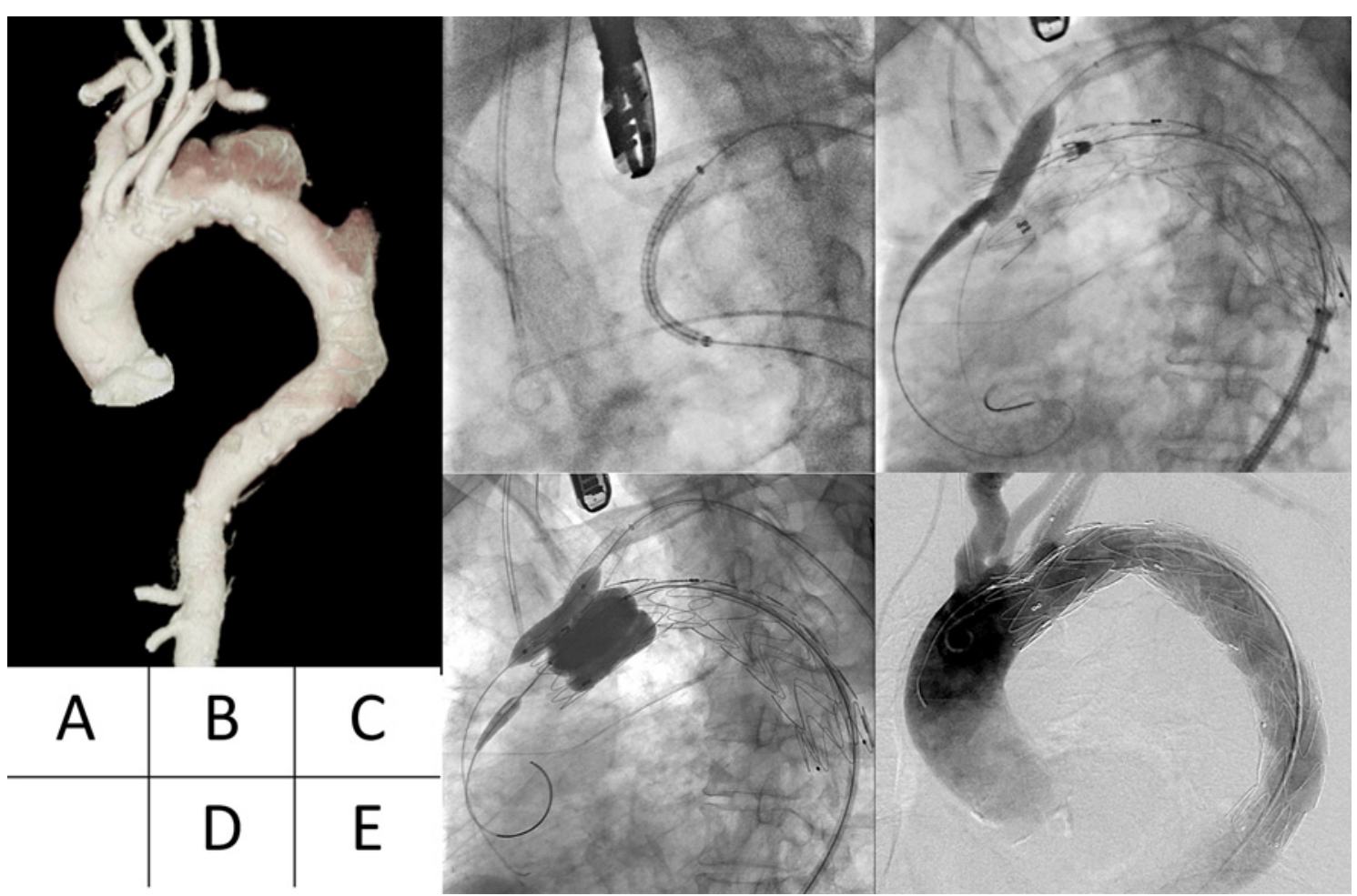

Figure 1. A) Computed tomography reconstruction demonstrates a saccular aneurysm of the distal aortic arch associated with a short proximal landing zone.

B) Fluoroscopic images obtained during the bidirectional approach of the chimney graft for downsizing of the chimney access. A covered stent was introduced from the left common femoral artery to the left subclavian artery over the brachiofemoral wire.

C) The covered stent was reoriented to the ascending aorta by balloon molding followed by deployment of the aortic stent-graft.

D) Kissing balloon molding in the chimney graft and aortic stent-graft was performed.

E) Digital subtraction angiography showed no evidence of an endoleak.

The diameter of the LSA was $11 \mathrm{~mm}$; therefore, a $13 \mathrm{~mm}$ covered stent requiring a 12-Fr introducer was adopted. We attempted a bidirectional approach of the chimney technique, which could be facilitated by the percutaneous brachial artery approach to avoid surgical exposure of the LSA (Figure 2).

The procedure was started with surgical cut-down of the left common femoral artery and percutaneous insertion of a 6-Fr introducer in the left brachial artery under general anesthesia. First, a covered stent (Viabahn; Gore, Flagstaff, AZ) was delivered to the LSA via the left common femoral artery over a brachiofemoral pull-through wire (Figure 1B). The covered stent was deployed in the LSA with a $>20-\mathrm{mm}$ proximal segment in the aortic lumen and approximately 20$\mathrm{mm}$ distal segment in the LSA (Figure 2A). Next, to execute the "reorientation of the chimney graft", the tip of the pull-through wire was repositioned into the ascending aorta through the left brachial artery access, and the covered stent was turned out into the ascending aorta using balloon dilatation (Admiral $10 \mathrm{~mm}-40 \mathrm{~mm}$, Medtronic, Minneapolis, MN, USA) (Figures 1C, 2B). In fact, the chimney graft spontaneously turned toward the ascending aorta after removing the pull-through wire. Care was taken to avoid involving the origin of the left common carotid artery when the proximal edge was reoriented into the ascending aorta. An aortic stent-graft (Valiant VAMF32-28-150; Medtronic) was then delivered via the left common femoral artery and deployed distal to the origin of the left common carotid artery, followed by kissing balloon angioplasty in the chimney graft and aortic stent-graft simultaneously (Tri-Lobe balloon, W. L. Gore \& Associates, Flagstaff, AZ, USA) (Figures 1D, 2 C). Angiography showed no evidence of an endoleak (Figure 1E). The fluoroscopic time was 47 minutes. No procedure-related complications occurred. Nonenhanced computed tomography images taken 1 year after the procedure demonstrated that the saccular aneurysms had slightly decreased.

\section{Discussion}

The first known chimney graft was implanted by Greenberg et al. in 1999 to rescue an overstented renal artery [2]. In 2002, Criado et al. reported the use of the chimney technique in TEVAR with a bare stent to rescue inadvertently covered LSAs [5]. The use of a covered stent in the chimney technique was first reported by Hiramoto et al. in 2006 


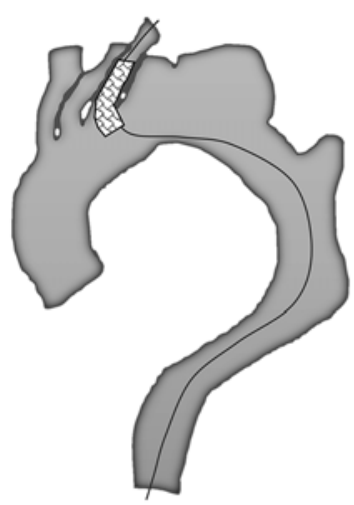

A) Deployment of the chimney graft

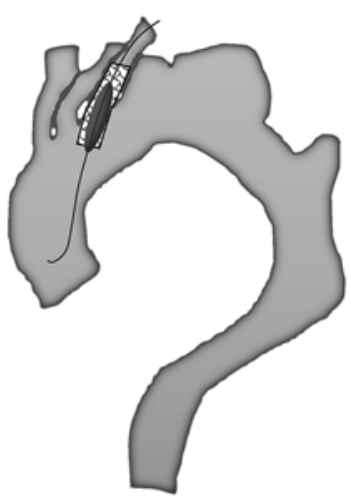

B) Repositioning of the chimney graft

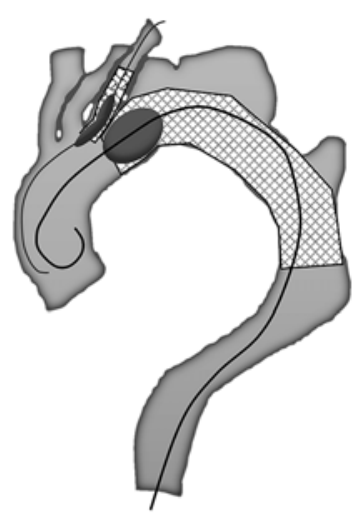

C) Kissing balloon molding

Figure 2. Methodology of the reorientation technique of the chimney graft for downsizing of the chimney access.

A) A covered stent was introduced from the left common femoral artery to the left subclavian artery over the brachiofemoral wire and deployed in the left subclavian artery with a $>20 \mathbf{m m}$ proximal segment in the aortic lumen.

B) The covered stent was reoriented to the ascending aorta by balloon molding.

C) Kissing balloon molding in the chimney graft and aortic stent-graft was performed after deployment of the aortic stent-graft.

to decrease the incidence of endoleaks [6]. The chimney technique using a self-expandable covered stent to maintain LSA perfusion is a safe treatment option for patients with aortic arch pathologies and insufficient proximal landing zones [3, 4]. LSA reconstruction is considered when the circle of Willis or vertebral artery is hypoplastic, stenotic, or occluded or there is a risk of spinal hypoperfusion due to long descending aorta coverage. The Society for Vascular Surgery practice guidelines recommended routine revascularization of the LSA when zone 2 TEVAR is necessary [7]. Revascularization is most commonly performed by a left carotid-subclavian bypass or transposition, but these operations have been associated with a 4-6\% stroke rate and 9$25 \%$ rate of nerve injury [8-10]. Axillo-axillary bypass may be considered as a suitable alternative and preferred when a concomitant ipsilateral carotid lesion is present, but the primary patency is up to $66 \%$ and can increase the complexity of future median sternotomy [11-13]. A recent large study demonstrated that endovascular LSA revascularization had lower risk of postoperative mortality, stroke, cardiac, and pulmonary complications compared to open LSA revascularization in the setting of Zone 2 TEVAR [14]. Compared with the hybrid technique, the chimney technique is more advantageous in terms of immediacy, reduced invasiveness, and improved safety [15]. Therefore, the present authors prefer the LSA chimney technique over open debranching.

Although indications and contraindications for chimney TEVAR remain unclear, chimney TEVAR has the potential risk of endoleaks and requires excess endograft oversizing and (kissing) balloon molding associating with retrograde thoracic aortic dissection. Therefore, if a patient has i) large $\left(>38^{\circ}\right)$ LSA angulation (because of type I endoleak, according to previous report by Zhao et al. [16]), ii) LSA length $<20 \mathrm{~mm}$ to vertebral artery (because of type II endoleak), iii) a proximal aortic landing zone diameter $>40 \mathrm{~mm}$ (because of retrograde thoracic aortic dissection), and iv) acute aortic dissection (due to retrograde thoracic aortic dissection), fenestrated TEVAR or hybrid TEVAR would be considered first. A type 3 arch, angulation, and tortuosity are not strictly prohibitive.

Endoleaks have been recognized as the major Achilles heel of the chimney technique because of channel gutters resulting from the apposition between the chimney graft and the aortic stent-graft. We used covered stents because they can reduce the rate of endoleaks to a certain extent [6]. Vascular access of a large-diameter covered stent is often impossible percutaneously and requires arteriotomy to insert an appropriately sized sheath. In the present case, the chimney technique with percutaneous brachial access was successfully performed by a bidirectional approach for delivery of the chimney graft. Notably, the chimney graft was advanced not from the brachial artery but from the femoral artery. Even chimney grafts requiring large-diameter access can be easily introduced using this technique. However, when the chimney graft is first deployed, its proximal edge faces toward the descending aorta. The chimney graft is then turned out into the ascending aorta by balloon dilatation via percutaneous brachial access. In fact, in this study, the chimney graft spontaneously turned toward the ascending aorta after removing the pull-through wire.

Lachat et al. reported on the chimney technique for pararenal abdominal aortic aneurysms using transfemoral access and the lift technique [17]. The lift technique may result in an even less invasive chimney TEVAR because brachial access is not required. However, the chimney graft can be dislodged from the branch artery during the "lift". Further- 
more, complex manipulation in diseased aortic arch would have an attendant risk of atheroembolization or retrograde aortic dissection.

To prevent gutter endoleaks, we also employed $30 \%$ oversized aortic stent-graft and $2 \mathrm{~cm}$ of overlap between the chimney graft and the thoracic stent-graft. Mesteres et al. found that better endograft stent apposition was usually attained when using 30\% oversizing during chimney technique [18]. A recent publication by Wang et al. recommended at least $2 \mathrm{~cm}$ of overlap between the chimney graft and the thoracic stent-graft to promote thrombosis in the gutter; additionally, the proximal end of the chimney graft should extend at least $0.5 \mathrm{~cm}$ beyond the proximal covered end of the aortic stent-graft [3]. If the proximal end of the migrated chimney graft extends beyond the proximal covered end of the aortic stent-graft only by $<0.5 \mathrm{~cm}$ or is compressed and deformed by the parallel aortic stent-graft, an additional bare stent could be delivered via percutaneous brachial access. Notably, however, the bare stent would not seal the vessel wall but would only provide a path for blood flow, which will eventually flow into the gutter and the proximal landing zone through the mesh of the bare stent, resulting in an endoleak [3].

As for the distal segment in the LSA, 10\% oversizing and $20 \mathrm{~mm}$ sealing would be deemed safe when using Viabahn. During withdrawing the chimney graft delivery system and advancement of a balloon catheter, care must be taken for dislocation of chimney graft.

The chimney technique has an $11 \%$ risk of a type I endoleaks due to the formation of so-called gutters and a $2 \%$ risk of occlusion of the chimney graft due to compression [3, 4]. In this bidirectional approach, if the position of the chimney graft after deployment is appropriate, the risk of endoleak formation and occlusion seem to be equivalent to that of the ordinary technique due to the use of a covered stent, overlapping of the grafts, and kissing balloon molding.

During the reorientation, the proximal edge of the chimney graft might end up in an unexpected and improper position. We recommend precise measurement and positioning of the chimney graft prior to the deployment of the thoracic aortic stent-graft. In addition, when deployed at a large curved vessel such as the thoracic aorta, migration of the chimney graft due to spring-back should also be taken into consideration. To solve this problem, we deployed the chimney graft with tightening the pull-through wire.

In conclusion, the bidirectional approach for delivery of the chimney graft could facilitate downsizing of left brachial artery access, even in cases involving a large-diameter chimney graft, when performing TEVAR with the chimney technique to preserve LSA flow.

\section{Funding: This study was not supported by any funding.}

Ethical approval: All procedures performed in studies involving human participants were in accordance with the ethical standards of the institutional and/or national research committee and with the 1964
Helsinki declaration and its later amendments or comparable ethical standards.

IRB statement: Institutional review board approval was waived.

Informed consent: Informed consent was obtained.

Consent for Publication: Consent for publication was obtained for every individual person's data included in the study.

Conflict of Interest: The authors have no conflict of interest to disclose.

Acknowledgement: We thank Angela Morben, DVM, ELS, from Edanz Group (www.edanzediting.com/ac), for editing a draft of this manuscript.

\section{References}

1. Hogendoorn W, Schlösser FJ, Moll FL, et al. Thoracic endovascular aortic repair with the chimney graft technique. J Vasc Surg 2013; 58(2): 502-511.

2. Greenberg RK, Clair D, Srivastava S, et al. Should patients with challenging anatomy be offered endovascular aneurysm repair? J Vasc Surg 2003; 38: 990-996.

3. Wang T, Shu C, Li M, et al. Thoracic Endovascular Aortic Repair With Single/Double Chimney Technique for Aortic Arch Pathologies. J Endovasc Ther 2017; 24: 383-393.

4. Pecoraro F, Lachat M, Cayne NS, et al. Mid-term Results of Chimney and Periscope Grafts in Supra-aortic Branches in High Risk Patients. Eur J Vasc Endovasc Surg 2017; 54: 295-302.

5. Criado FJ, Barnatan MF, Rizk Y, et al. Technical strategies to expand stent-graft applicability in the aortic arch and proximal descending thoracic aorta. J Endovasc Ther 2002; 9: II32-II38.

6. Hiramoto JS, Schneider DB, Reilly LM, et al. A double-barrel stent-graft for endovascular repair of the aortic arch. J Endovasc Ther 2006; 13: 72-76.

7. Matsumura JS, Lee WA, Mitchell RS, et al. The society for vascular surgery practice guidelines: management of the left subclavian artery with thoracic endovascular aortic repair. J Vasc Surg 2009; 50: 1155.

8. Schardey HM, Meyer G, Rau HG, et al. Subclavian carotid transposition: an analysis of a clinical series and a review of the literature. Eur J Vasc Endovasc Surg 1996; 12: 431-436.

9. Cinà CS, Safar HA, Laganà $\mathrm{A}$, et al. Subclavian carotid transposition and bypass grafting: consecutive cohort study and systematic review. J Vasc Surg 2002: 35; 422-429.

10. Voigt SL, Bishawi M, Ranney D, et al. Outcomes of carotidsubclavian bypass performed in the setting of thoracic endovascular aortic repair. J Vasc Surg 2019; 69: 701-709.

11. AbuRahma AF, Robinson PA, Khan MZ, et al. Brachiocephalic revascularization: a comparison between carotid-subclavian artery bypass and axilloaxillary artery bypass. Surgery 1992; 112: 84-91.

12. Mingoli A, Feldhaus RJ, Farina C, et al. Comparative results of carotid-subclavian bypass and axillo-axillary bypass in patients with symptomatic subclavian disease. Eur J Vasc Surg 1992; 6: 26-30.

13. Perkins JM, Magee TR, Hands LJ, et al. The long-term outcome after axillo-axillary bypass grafting for proximal subclavian artery disease. Eur J Vasc Endovasc Surg 2000; 19: 52-55.

14. Delafontaine JL, Hu B, Tan TW, et al. Outcome Comparison of TEVAR with and without Left Subclavian Artery Revascularization from Analysis of Nationwide Inpatient Sample Database. Ann 
Vasc Surg 2019; 58: 174-179.

15. Ding H, Liu Y, Xie N, et al. Outcomes of Chimney Technique for Preservation of the Left Subclavian Artery in Type B Aortic Dissection. Eur J Vasc Endovasc Surg 2019; 57: 374-381.

16. Zhao Y, Cui J, Yin H, et al. Hybrid operation versus the chimney technique for reconstruction of a single aortic arch branch: asingle-centre experience. Interact Cardiovasc Thorac Surg. 2017; 25: 575-581.

17. Lachat M, Bisdas $T$, Rancic Z, et al. Chimney endografting for pararenal aortic pathologies using transfemoral access and the lift technique. J Endovasc Ther 2013; 20: 492-497.

18. Mestres G, Yugueros $X$, Apodaka A, et al. The best in vitro conditions for two and three parallel stenting during endovascular aneurysm repair. J Vasc Surg 2017; 66: 1227-1235.

Interventional Radiology is an Open Access journal distributed under the Creative Commons Attribution-NonCommercial 4.0 International License. To view the details of this license, please visit (https://creativecommons.org/licenses/by$\mathrm{nc} / 4.0 /)$. 